\title{
ELASTICITY AND VISCOSITY OF DNA LIQUID CRYSTALS
}

\author{
SUPPORTING INFORMATION
}

Liana Lucchetti ${ }^{1}$, Tommaso P. Fraccia ${ }^{2,3}$, Giovanni Nava ${ }^{1,3}$, Taras Turiv ${ }^{4}$, Fabrizio Ciciulla ${ }^{1}$, Lucas Bethge $^{5}$, Sven Klussmann ${ }^{5}$, Oleg D. Lavrentovich ${ }^{4,6}$, Tommaso Bellini, ${ }^{3, *}$

1. Dipartimento SIMAU, Università politecnica delle Marche, via Brecce Bianche, 60131 Ancona, Italy

2. Institute Pierre-Gilles de Gennes, CBI UMR8231, ESPCI Paris-CNRS, PSL Research University, 6 rue Jean Calvin, 75005, Paris, France

3. Dipartimento di Biotecnologie Mediche e Medicina Traslazionale, Università di Milano, via Fratelli Cervi 93, I-20090 Segrate (MI), Italy

4. Advanced Materials and Liquid Crystal Institute, Chemical Physics Interdisciplinary Program, Kent, OH 44242, USA Department of Physics, Kent State University, Kent, Ohio 44242, USA

5. NOXXON Pharma AG, 10589 Berlin, Germany

6. Department of Physics, Kent State University, Kent, Ohio 44242, USA

* Corresponding author: tommaso.bellini@unimi.it

\section{Preparation of the plates with photoaligned multilayer coating}

The uniform alignment of DNA Nematic Liquid Crystal (NLC) is achieved by the multilayer structure of a photosensitive azo-dye and passivation layer of a liquid crystal elastomer. The azo-dye is a photoalignment agent known to align the director of thermotropic liquid crystals perpendicularly to linear polarization of light [1]. The alignment stability is often enhanced by a layer of a liquid crystal elastomer [2,3]. Such a layer is of importance as a physical barrier in the alignment of water-based materials such as lyotropic chromonic liquid crystals and DNA LCs, since the underlying layer of azobenzene dye is water soluble. In our alignment of DNA LC we followed the procedure developed previously for lyotropic chromonic liquid crystals [4], as detailed below.

We use soda lime glass slides of rectangular shape $(15 \mathrm{~mm} \times 12 \mathrm{~mm}$ lateral size $)$ and thickness of $1 \mathrm{~mm}$. The glass is cleaned in the ultrasonic bath of deionized water and small concentration of detergent at $65^{\circ} \mathrm{C}$ for 20 minutes. We rinse the glass with deionized water and isopropanol, let it dry for $10 \mathrm{~min}$ at $85^{\circ} \mathrm{C}$ and further cleaned inside the UV-ozone chamber for 15 minutes to remove any remaining organic contamination and improve the wettability properties. Immediately after the glass is spin-coated with solution of $0.3 \mathrm{wt} \%$ azo-dye Brilliant Yellow (Sigma-Aldrich), see Fig. S1 A for the details of chemical structure, in dimethyl formamide at $3000 \mathrm{rpm}$ for 30 seconds. After spin-coating the substrate is annealed inside the oven at $100^{\circ} \mathrm{C}$ for 30 minutes. We then illuminate the glass with metal-halide X-Cite 120 lamp through linear polarizer to induce the easy axis of alignment which is perpendicular to the linear polarization of light. The illumination is performed for 5 minutes. The glass with azo-dye coating is spin-coated with $6.7 \mathrm{wt} \%$ LC diacrylate monomer RM257 (Wilshire Technologies), Fig. S1 B, and $0.3 \mathrm{wt} \%$ of photoinitiator Irgacure 651 (Ciba Specialty Chemicals Inc.), Fig. S1 C, in toluene at $3000 \mathrm{rpm}$ for $30 \mathrm{sec}$. Then it is immediately placed under the $365 \mathrm{~nm}$ UV light of UVP-58 lamp for 15 minutes (light intensity $1.8 \mathrm{~mW} \mathrm{~cm}^{-2}$ ) at room temperature and ambient atmosphere to fully polymerize the diacrylate monomer. The thickness of the resulting aligning layers is 150 $\mathrm{nm}$ and their birefringence $\Delta \mathrm{n}_{\mathrm{sb}} \approx 0.18$. 
Two substrates with photoaligned and photopolymerized liquid crystal polymer are then assembled to form flat thin cells enclosing rDNA NLC. The two substrates are oriented so that the photoalignment axes are parallel to each other and held at a distance of $\mathrm{L}=15 \mu \mathrm{m}$ by silica spacers. rDNA NLC is then sealed by being surrounded by fluorinated oil. Fluorinated oil - perfectly transparent and isotropic - is filling the region in the upper right corner - top panel - and in the lower right corner - bottom panel - of Fig. 1 of the main text. The difference in transmittance of that region between the two panels is due to the small but noticeable birefringence of the photopolymerized LC polymer layer on the substrates. The optical shift for a single substrate is $\zeta_{\text {ab }} \approx 30 \mathrm{~nm}$, a value that should be compared to the optical shift $\zeta \approx-400 \mathrm{~nm}$ due to the DNA LC.

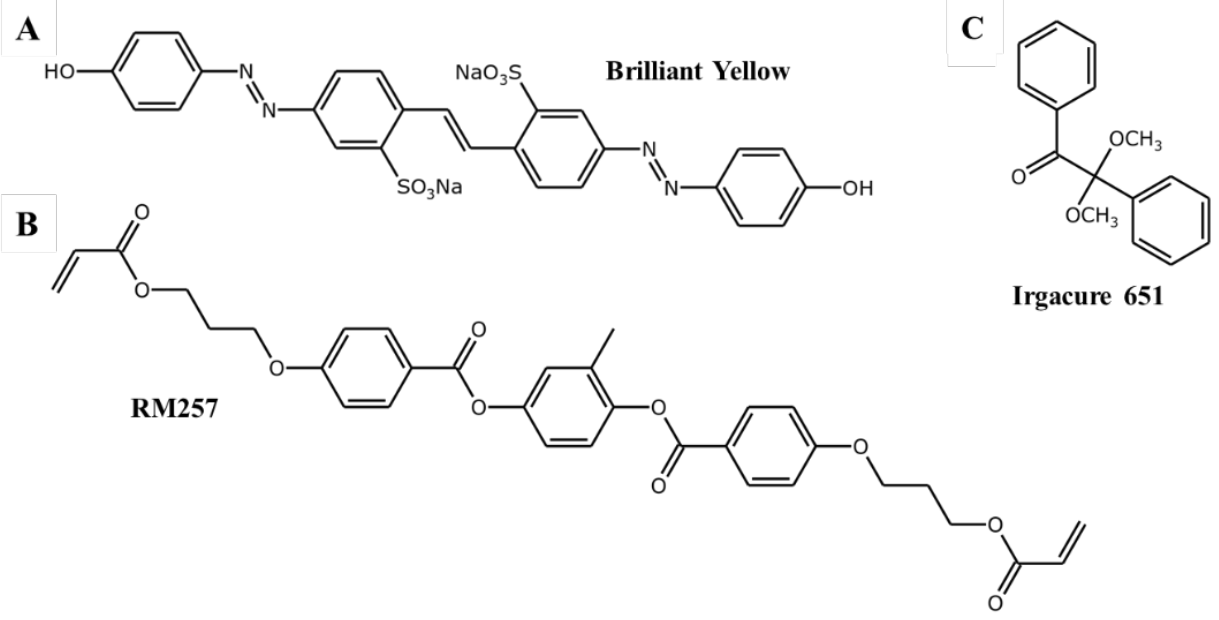

Figure S1. Chemical structures of substances used. Chemical structure of (A) photoaligning azo-dye Brylliant Yellow, (B) diacrylate monomer RM257, and (C) photoinitiator Irgacure 651.

\section{Expression of the optical torque in the S.I. units}

In the following we will make use of the International System of units. In this frame, the optical field E and light beam intensity I are related as

$$
E^{2}=\frac{2}{\epsilon_{0}} \frac{I}{c n}
$$

while the optical torque is

$$
\Gamma=\boldsymbol{P} \times \boldsymbol{E}=\frac{1}{2} \epsilon_{0} \Delta \epsilon_{r} E^{2} \cos \beta \sin \beta
$$

where the factor $1 / 2$ comes from the time average of the oscillating field. $\varepsilon_{0}$ is the dielectric permittivity of vacuum and $\Delta \varepsilon_{\mathrm{r}}$ the dielectric anisotropy of the material at optical frequency. $\beta$ is the angle between the optical field and the axis of the polarizable material.

\section{Computation of the splay deformation induced by optical torque (Geometry A)}

In geometry A the pump beam is tilted with respect to the layer normal by an angle $\beta$. Both the direction of the pump beam and its polarization lay in the x-z plane, as shown in Fig. 2. A1 and A2 of the main text. The optical field acting on the NLC is thus also tilted by an angle $\beta$ with respect to the $\mathrm{z}$ direction and is uniform in the illuminated region. As a consequence, the director experiences a torque $\Gamma$, and tilts by an angle $\vartheta(z)$ within the $\mathrm{x}-\mathrm{z}$ plane. In equilibrium, the optical torque density 


$$
\Gamma=\frac{\Delta n}{c} \mathrm{I}_{\mathrm{p}} \sin 2 \beta
$$

and the splay elastic torque density

$$
\Gamma_{E L}=K_{1} \frac{d^{2} \vartheta}{d z^{2}}
$$

are balanced, so that

$$
\frac{d^{2} \vartheta}{d z^{2}}=\frac{\Delta n I_{p} \sin 2 \beta}{c K_{1}}
$$

In $\Gamma_{E L}$ we have adopted the approximation $\vartheta(\mathrm{z})<<1$. Since $\vartheta(0)=\vartheta(\mathrm{L})=0$ because of the coupling with the surface, Eq. S3 leads to

$$
\vartheta(\mathrm{z})=\frac{\Delta n I_{p} \sin 2 \beta}{2 c K_{1}} z(L-z)
$$

From the tilt angle profile $\vartheta(\mathrm{z})$ we can compute the effective order parameter experienced by light travelling through the cell at an angle $\beta$ and thus the difference in optical path for optical fields polarized in the $x-y$ and $\mathrm{x}$-z planes. The phase shift $\delta$ produced between the two is

$$
\delta=\delta_{0}\left(\frac{3 \cos \beta-1}{2}+\frac{\Delta n I_{p} L^{2} \sin ^{2} 2 \beta}{8 c K_{1}}\right) \frac{1}{\cos \beta}
$$

where $\delta_{0}=2 \pi L \Delta n / \lambda$. In practice, because of the additional birefringence of the aligning layers, the unperturbed phase shift experienced by the light while crossing the cell is $\delta_{0}{ }^{\prime}=\delta_{0}+\delta_{\mathrm{ab}}$, with $\delta_{\mathrm{ab}}=2 \pi \zeta_{\mathrm{uu}} / \lambda$. Both in this and in the next geometry, the effect of the birefringence of the aligning layers in the evaluation of the elastic coefficients can be approximated by replacing $\delta_{0}$ with $\delta_{0}$ '.

From Eq. S5 we can compute the probe light intensity transmitted between cross polarizers in Geometry A $\left(I_{1}\right)$, normalized for the probe intensity $\mathbf{I}_{0}$

$$
\frac{I_{1}}{I_{0}}=\sin ^{2}\left(\frac{\delta}{2}\right)=\sin ^{2}\left(\frac{\delta_{0}^{\prime}}{2 \cos \beta}\left(\frac{3 \cos ^{2} \beta-1}{2}+\frac{\Delta n I_{P} L^{2} \sin ^{2} 2 \beta}{8 c K_{1}}\right)\right)
$$

By expanding for small $I_{P}$, i.e. at the lowest order in the perturbation of the nematic director, we obtain

$$
\frac{I}{I_{0}}=\sin ^{2}\left(\delta_{0}^{\prime} \frac{3 \cos \beta-1}{4 \cos \beta}\right)+\delta_{0}^{\prime} \frac{\Delta n I_{P} L^{2}}{16 c K_{1}} \frac{\sin ^{2} 2 \beta}{\cos \beta} \sin \left(\frac{\delta_{0}^{\prime}}{\cos \beta}\left(\frac{3 \cos \beta-1}{2}\right)\right)
$$

This equation indicates that, to a first order, the change in the transmitted intensity is linear in the pump intensity, i.e. it has the shape

$$
\frac{I_{1}}{I_{0}}=A_{1}+\frac{B_{1}}{K_{1}} I_{P}
$$

where the coefficients $A_{1}$ and $B_{1}$ depend on the experimental design $(L, \Delta n, \lambda, \beta)$ : 


$$
\begin{gathered}
A_{1}=\sin ^{2}\left(\delta_{0}^{\prime} \frac{3 \cos \beta-1}{4 \cos \beta}\right) \\
B_{1}=\frac{\delta_{0}^{\prime} \Delta n L^{2}}{16 c} \frac{\sin ^{2} 2 \beta}{\cos \beta} \sin \left(\frac{\delta_{0}^{\prime}}{\cos \beta}\left(\frac{3 \cos \beta-1}{2}\right)\right)
\end{gathered}
$$

\section{Computation of the twist deformation induced by optical torque (Geometry B)}

Geometry B is designed to induce a deformation of pure twist. This is described in Fig. 2, B1 and B2 of the main text. We illuminate the sample with both pump and probe perpendicular to the cell surface. The polarization of the pump is oriented at $45^{\circ}$ with respect to the rubbing direction, thus producing a torque in the $\mathrm{x}-\mathrm{y}$ plane. In the thin cell limit, the optical torque on the director is uniform throughout the cell and is equal to

$$
\Gamma_{\text {thin }}=\frac{\Delta n}{c} \mathrm{I}_{\mathrm{p}}
$$

which is balanced by the twist elastic torque density

$$
\Gamma_{E L}=K_{2} \frac{d^{2} \varphi}{d z^{2}}
$$

where $\varphi$ is the angle between director and rubbing direction in the $x-y$ plane. Boundary conditions are $\varphi(0)=$ $\varphi(\mathrm{L})=0$. In the thin cell limit we obtain

$$
\varphi(\mathrm{z})=\frac{\Delta n I_{p}}{2 c K_{2}} z(L-z)
$$

This deformation changes the optical properties of the cell in two ways: (i) by disrupting the uniform alignment, it induces a decrease of the effective birefringence; (ii) by favoring a different direction of the director in the xy plane, it modifies the average direction of the optical axis. These two effects can be explicitly calculated by computing the 2D nematic order parameter tensor on the basis of the angular distribution of the nematic director. From its diagonalization we obtain that the tilt of the average optical axis $\langle\varphi\rangle$ is

$$
\langle\varphi\rangle=\frac{\Delta n I_{p}}{12 K_{2}}
$$

while from its eigenvalues we find that the change in the effective birefringence is $\delta \Delta n=\mathrm{O}\left(E^{4}\right)$ and thus can be neglected when the twist is small.

The change in the optical axis can be detected by the probe beam, which propagates counter to the pump beam with polarization at an angle $\alpha$ with respect to the rubbing direction. The probe light is measured after an analyzer oriented perpendicularly to its initial polarization $(\alpha-\pi / 2)$. We have performed experiments with $\alpha=35^{\circ}$ and $\alpha=55^{\circ}$. The choice $\alpha=45^{\circ}$, which maximizes transmission, is not appropriate since it minimizes the sensitivity to small tilts of the optical axis.

In these conditions the transmitted intensity of the probe is

$$
\frac{I_{2}}{I_{0}}=\sin ^{2}\left(\frac{\delta}{2}\right) \sin ^{2}(2(\alpha-\langle\varphi\rangle)) \approx \sin ^{2}\left(\frac{\delta_{0}^{\prime}}{2}\right) \sin ^{2}(2 \alpha)-\frac{\Delta n I_{P} L^{2} \sin (4 \alpha) \sin ^{2}\left(\delta_{0}^{\prime} / 2\right)}{6 c K_{2}}
$$


It is worth pointing out that this result relies on two basic simplifications: (i) we assume that the main effect of the field-induced twist on the probe beam propagation can be approximated by rigidly rotating the unperturbed cell by $\langle\varphi\rangle$; (ii) we consider only effects to the lowest order in pump power.

This result applies in the limit of thin cells, where the polarization of the pump beam is uniform throughout the cell. When instead the difference of optical path of ordinary vs. extraordinary polarization is not much smaller than the wavelength, the change in the polarization state as the pump beam propagates through the cell should be taken into account. This is the case of the experiments described here in which $\Delta n=0.025$ (rDNA LC) and $\Delta n=0.03\left(\mathrm{CCN} 47\right.$ at $\left.\mathrm{T}=40^{\circ} \mathrm{C}\right)$, while the cell thickness are $\mathrm{L} \approx 15 \mu \mathrm{m}$ and $\mathrm{L} \approx 10 \mu \mathrm{m}$, respectively. This corresponds to phase shifts of $\delta \approx 0.6 \times 2 \pi$ (DNA LC) and $\delta \approx 0.5 \times 2 \pi$ (CCN47), which should most definitely be taken into account since it implies that the polarization of the pump is heavily modified as it travels across the cell.

The dependence of the pump polarization on the z-coordinate can be taken into account by explicitly including a $\mathrm{z}$ dependence of $\Gamma$. Accordingly, we assume

$$
\Gamma(z)=\frac{\Delta n I_{P}}{c} \cos (2 \pi \Delta n z / \lambda)
$$

The equilibrium twist profile can be still be computed by the balance of elastic and optical torques:

$$
\varphi(\mathrm{z})=\frac{I_{P} \lambda_{P}^{2}}{4 \pi^{2} c \Delta n L K_{2}}\left(L-z+z \cos \frac{2 \pi \Delta n L}{\lambda_{p}}-L \cos \frac{2 \pi \Delta n z}{\lambda_{p}}\right)
$$

so that the transmitted intensity, evaluated in the same approximations adopted for thin cells above, becomes

$$
\frac{I}{I_{0}} \approx \sin ^{2}\left(\frac{\delta_{0}^{\prime}}{2}\right) \sin ^{2}(2 \alpha)-\frac{\Delta n I_{P} \mathrm{~L}^{2} \sin (4 \alpha) \sin ^{2}\left(\delta_{0}^{\prime} / 2\right)}{6 c K_{2}} \frac{3 \lambda_{P}^{3}}{2 \pi^{3} \Delta n^{3} L^{3}}\left[\frac{\Delta n L \pi}{\lambda_{p}}\left(1+\cos \frac{2 \pi \Delta n L}{\lambda_{p}}\right)-\sin \frac{2 \pi \Delta n L}{\lambda_{p}}\right]
$$

where the last term in square brackets is an effective correction to the result for thin cells (Eq. S15) arising because of the $\mathrm{z}$ dependence of the optical torque. The effect of the cell thickness is to introduce an effective thickness approximately equal to $\lambda_{p} / \pi \Delta n$ which dominates over the real thickness $\mathrm{L}$.

Therefore, the intensity transmitted through polarizers has the shape

$$
\frac{I_{2}}{I_{0}}=A_{2}+\frac{B_{2}}{K_{2}} I_{P}
$$

where

$$
\begin{gathered}
A_{2}=\sin ^{2}\left(\frac{\delta_{0}^{\prime}}{2}\right) \sin ^{2}(2 \alpha) \\
B_{2}=-\frac{\Delta n \mathrm{~L}^{2} \sin (4 \alpha) \sin ^{2}\left(\delta_{0}^{\prime} / 2\right)}{6 c} \frac{3 \lambda_{P}^{3}}{2 \pi^{3} \Delta n^{3} L^{3}}\left(\frac{\Delta n L \pi}{\lambda_{p}}\left(1+\cos \frac{2 \pi \Delta n L}{\lambda_{p}}\right)-\sin \frac{2 \pi \Delta n L}{\lambda_{p}}\right)
\end{gathered}
$$

It is worth noticing that the correction for thick cell is not relevant in Geometry A, since in that case the pump was polarized in the $\mathrm{x}-\mathrm{y}$ plane, a symmetry plane in the index ellipsoid, and thus does not modify its polarization state. 


\section{Viscoelastic relaxation in Geometry B}

The kinetic response of the nematic ordering to the application and removal of the optical field $\varphi(\mathrm{z}, \mathrm{t}) \mathrm{can}$ be evaluated by solving the nematodynamic equations $\gamma \partial_{t} \varphi_{o n}(x, t)=K_{2} \partial_{z}^{2} \varphi_{o n}(x, t)+\tau_{O P T}$ with $\varphi_{\text {on }}(\mathrm{z}, 0)=0$ for the "on" response after switching on the pump field, and $\gamma \partial_{t} \varphi_{\text {off }}(x, t)=K_{2} \partial_{z}^{2} \varphi_{\text {off }}(x, t)$ with $\varphi_{\text {off }}(\mathrm{z}, 0)=\varphi(\mathrm{z})$ for the "off" relaxation after turning off $\mathrm{I}_{\mathrm{p}}$. The equations can be solved in both cases, the solution being of the format

$$
\begin{aligned}
& \varphi_{\mathrm{on}}(\mathrm{z}, 0)=\sum_{m \geq 1} k_{m}\left(1-e^{-t / \tau_{m}}\right) f_{m}(z) \\
& \varphi_{\mathrm{off}}(\mathrm{z}, 0)=\sum_{m \geq 1} k_{m} e^{-t / \tau_{m}} f_{m}(z)
\end{aligned}
$$

where $k_{m}$ are coefficients in the expansion, $f_{m}(z)=\sin (m \pi z / L)$ are the Fourier components $\left(f_{m}(0)=\right.$ $\left.f_{m}(L)=0\right)$, and $\tau_{m}=\gamma L^{2} / m^{2} \pi^{2} K_{2}$.

Eqs. S22 and S23 indicate that the on and off relaxations have equal kinetics, as is expected, and that they are both non-exponential. Inspection of the equilibrium and kinetic solutions evaluated in the regime of parameters of interest to this work indicate that the solutions truncated to $m=2$ are good approximations to the full solution of $\varphi(\mathrm{z}, \mathrm{t})$, with deviation always less than $5 \%$ of its value. As a consequence, any quantity that linearly depends on $\varphi(\mathrm{z}, \mathrm{t})$ is expected to be well represented by a double exponential kinetics, with characteristic times $\tau_{1}=\gamma L^{2} / \pi^{2} K_{22}$ and $\tau_{2}=\gamma L^{2} / 4 \pi^{2} K_{22}$. This observation is here of relevance because it enables us to analyze the kinetic data in a rather simple way. Since the field induced distortions are small (they never exceed $3^{\circ}$ ), the propagation of the probe beam through the twisted profile of the cell must be a linear functional of $\varphi(\mathrm{z}, \mathrm{t})$. We thus analyzed the measured off relaxations, of which experiments enabled much longer acquisition times than for on relaxations, as a sum of two exponentials of undetermined amplitude, in which we constrain $\tau_{2}=\tau_{1} / 4$. The fits are very good, as shown in Fig. 2.B3 of the main text. When instead the same relaxations are fitted with a single exponential, the agreement is poor, clearly indicating the presence of multiple characteristic times. The parameters obtained from the off data can be used to fit, with very good agreement, the on data as well.

\section{Computation of the bend deformation induced by optical torque (Geometry C)}

In Geometry $\mathrm{C}$ we produced a polarization grating on the DNA NLC cell. This is obtained by superimposing two beams of opposite circular polarization that converge on the cell at an angle $\Omega$ (see Fig. 2.C1 of the main text). This produces a periodic rotation of the linear polarization at constant intensity. In other words, the field in the overlap region is linearly polarized, with the direction of the polarization $\beta$ (referred to the $\mathrm{x}$ axis, i.e. the rubbing direction) that does not depend on $\mathrm{z}$ but grows linearly with $\mathrm{x}$ as $\beta=\pi x / \Lambda$. The linearly polarized field exerts a torque on the nematic director

$$
\Gamma=\frac{\Delta n I_{P}}{c} \sin \frac{2 \pi x}{\Lambda}
$$

As in the determination of the elastic coefficients above, here also the birefringence of the aligning layers produces a small effect which could be taken into account. The birefringent aligning layer acts as a thin phase shifter slightly modifying the polarization state of the two interfering beams. Its effect can be computed and taken into account as an effective reduction of the torque $\Gamma$. Such reduction can be expressed as $\Gamma^{\prime}=h \Gamma$, where $\mathrm{h} \approx 0.97$.

This torque acts to tilt the director away from $\mathrm{x}$ in the $\mathrm{x}-\mathrm{y}$ plane periodically in $\mathrm{x}$ but uniformly in $\mathrm{z}$, as sketched in Fig. 2.C2 of the main text. This $\varphi(x)$ perturbation corresponds to a pure bend deformation. However, while the torque is uniform in $\mathrm{z}$, the boundary conditions $\varphi(0)=\varphi(\mathrm{L})=0$ induce a $\mathrm{z}$ dependence of 
the solution, which can thus be obtained by solving

$$
\frac{\Delta n}{c} \mathrm{I}_{\mathrm{p}} \sin \frac{2 \pi x}{\Lambda}=K_{2} \frac{d^{2} \varphi(\mathrm{x}, \mathrm{z})}{d z^{2}}-K_{3} \frac{4 \pi^{2}}{\Lambda^{2}} \varphi(\mathrm{x}, \mathrm{z})
$$

While the full solution can be computed from Eq. S25, it is worth noticing that, in our experimental conditions, the dominant term on the right-hand side of Eq. S25 is the last one. This is because the pitch of the grating, the length-scale for bend deformation, is shorter than the cell thickness over which the twist deformation develops, i.e. $\Lambda \sim 3-5 \mu \mathrm{m}<<\mathrm{L} \approx 15 \mu \mathrm{m}$. Moreover, the elastic coefficient $\mathrm{K}_{3}$ is generally, and also in our case, larger than $\mathrm{K}_{2}$. Therefore, Eq. S25 can be simplified into

$$
\frac{\Delta n}{c} \mathrm{I}_{\mathrm{p}} \sin \frac{2 \pi x}{\Lambda} \approx-K_{3} \frac{4 \pi^{2}}{\Lambda^{2}} \varphi(\mathrm{x}, \mathrm{z})
$$

from which

$$
\varphi(\mathrm{x}, \mathrm{z}) \approx-\frac{\Delta n \mathrm{I}_{\mathrm{p}} \Lambda^{2}}{4 \pi^{2} c K_{3}} \sin \frac{2 \pi x}{\Lambda}
$$

Because of the periodic bend of the optical axis, the deformed cell is effectively a phase grating. Thus, the impinging beams are self-diffracted, as we indeed observe. This is an advantageous condition, since the diffracted intensity can be exploited as a probe for the amplitude of the nematic deformation. Thus, we need to evaluate the diffraction efficiency from the elastic description of the system.

To do this, let's consider one of the two pump beams and evaluate the difference in its polarization state as it exits the cell in the two conditions of: 1) undistorted ordering $(\varphi=0)$ and 2$)$ director uniformly tilted by $\varphi_{\mathrm{M}}=\frac{\Delta n \mathrm{I}_{\mathrm{p}} \Lambda^{2}}{4 \pi^{2} c K_{3}}$, i.e. the maximum deformation in Eq. S27. The difference between the two optical fields (let's call them $\mathrm{E}_{1}$ and $\mathrm{E}_{2}$ ) can be computed with the aid of Jones matrices. We obtain that the difference in amplitude between the field is

$$
\delta E=E_{2}-E_{1} \approx E_{p} 2 \sin \frac{\pi \Delta n L}{\lambda_{v}} \varphi_{\mathrm{M}} \sin \frac{2 \pi x}{\Lambda}
$$

while it depends on time as a circularly polarized field. $E_{\mathrm{p}}$ in (S28) is the pump optical field. The amount $\delta \mathrm{E}$ expresses the amplitude of the modulation in the outcoming light when the grating is present. As such, it also gives the amplitude of the diffracted beam

$$
I_{d}=\frac{\epsilon_{0} c n}{2}|\delta E|^{2}=I_{p}\left(2 \sin \frac{\pi \Delta n L}{\lambda_{v}}\right)^{2} \phi_{M}^{2}
$$

Thus, the dependence of the diffracted intensity on the pump intensity has the shape

$$
I_{d}=\frac{C_{3}}{K_{3}^{2}} I_{p}^{3}
$$

where

$$
C_{3, \text { thin }}=\frac{\Delta n^{2} \Lambda^{4}}{4 \pi^{4} c^{2}} \sin ^{2} \frac{\pi \Delta n L}{\lambda_{v}}
$$

In practice, even in this geometry, the evaluation is actually more complex because of two factors:(i) as in the study of $\mathrm{K}_{2}$, the thickness of the cell needs to be considered. As a consequence, the polarization of the two beams changes as they travel across the cell, thus modifying along $\mathrm{z}$ the torque they exert. In close analogy to 
what discussed in the case of $\mathrm{K}_{2}$, this implies a $\mathrm{z}$ dependence of $\varphi$, which becomes

$$
\varphi(\mathrm{x}, \mathrm{z}) \approx-\frac{\Delta n \mathrm{I}_{\mathrm{p}} \Lambda^{2}}{4 \pi^{2} c K_{3}} \sin \frac{2 \pi x}{\Lambda} \cos \frac{2 \pi \Delta n z}{\lambda}
$$

(ii) the beams propagate in the cell at an angle $\Omega / 2$, so that the values $\varphi$ encountered by the light travelling through the cell is not simply $\varphi(\mathrm{z})$.

To take into account both phenomena, we evaluated the diffraction efficiency by replacing $\phi_{M}$ in Eq. S29 with $\langle\varphi\rangle$, i.e. the average tilt of the optical axis experienced by the diffracted beam

$$
\langle\varphi\rangle=\frac{1}{L} \int_{S} \varphi(\mathrm{x}(\mathrm{s}), \mathrm{z}(\mathrm{s})) d s
$$

where $\mathrm{S}$ is the path along the direction of the beam and $\mathrm{x}(\mathrm{s})$ and $\mathrm{z}(\mathrm{s})$ are the coordinates along the path. By computing the $2 \mathrm{D}$ order parameter from the distribution of $\varphi$ experienced by oblique rays (of angle $\Omega / 2$ ) entering the cell in every $\mathrm{x}$ position, and by diagonalizing it and determining the eigenvalues (effective birefringence) and eigenvectors (effective optical axis) as a function of $\mathrm{x}$, we obtain

$$
\langle\varphi\rangle=\frac{\Delta n \mathrm{I}_{\mathrm{p}} \Lambda^{2}}{4 \pi^{2} c K_{3}} B_{3}(\Omega, \Delta n, \lambda, L) \sin \frac{2 \pi\left(x+\delta_{3}(\Omega, \Delta n, \lambda, L)\right)}{\Lambda}
$$

where $\mathrm{B}_{3}$ and $\delta_{3}$ are two parameters depending on $\Omega, \Delta n, \lambda$ and $\mathrm{L}$ that need to be evaluated numerically. Eq. S34 indicates that the inclusion of the effects due to the cell thickness and oblique propagation amount to a multiplicative parameter $\left(\mathrm{B}_{3}\right)$ that reduces the amplitude of the modulation experienced by light and a phase that shifts the position of the polarization grating along the $\mathrm{x}$ axis. This latter effect has no relevance on the efficiency of self-diffraction. Thus, overall, the system retains the shape in Eq. S30 with

$$
C_{3}=\frac{\Delta n^{2} \Lambda^{4}}{4 \pi^{4} c^{2} K_{3}^{2}} \sin ^{2} \frac{\pi \Delta n L}{\lambda_{v}} B_{3}(\Omega, \Delta n, \lambda, L)
$$

\section{Experimental determination of the diffracted intensity}

As clearly visible in Fig.2 C3 of the main text, the intensity $I_{c}$ that we measure in the location where the first order diffraction is expected depends on time with an abrupt increase - compatible with the time of the opening of the shutter controlling the pump beam and having an amplitude proportional to $I_{p}$, followed by a small mild increase that can be well approximated by an exponential. When the intensity is measured outside the area of the diffracted beam, we observe a stepwise increase of similar amplitude with no subsequent relaxation. These observations indicate that $I_{c}$ is actually obtained by adding two different contributions, the expected diffracted light $I_{d}$, and the light $I_{s}$ scattered by surface roughness, impurities, and static and dynamic distortions in the systems - proportional to the illuminating light and of amplitude equal to the step increment of $I_{c}$. The two contributions can be distinguished by evaluating the mixing effect of the scattered and diffracted optical fields

$$
I_{C}=\frac{\epsilon_{0} c n}{2}\left|E_{d}+E_{s}\right|^{2}=I_{d}+I_{s}+\epsilon_{0} c n \operatorname{Re}\left(E_{d}^{*} E_{s}\right) \approx I_{s}+2 \sqrt{I_{s} I_{d}}
$$

and thus 


$$
I_{d}=\frac{\left(I_{C}-I_{S}\right)^{2}}{4 I_{S}}
$$

Examples of values of $\mathrm{I}_{d}$ obtained in this way are shown Fig.2 C4 and C5 of the main text. The fitted lines indicate that we find $\mathrm{I}_{d} \propto \mathrm{I}_{\mathrm{p}}{ }^{3}$ as expected on the basis of Eq. S30. This behavior confirms that the extraction method in Eq. S33 is correct.

\section{Elasticity and viscosity of thermotropic liquid crystals}

The same approach used to measure the viscoelasticity of DNA LC was also applied to the thermotropic LC 5CB (in Geometry 1 - splay), CCN47 (in Geometry 1 - splay and Geometry 2 - twist) and CB7CB (Geometry 3 - bend) and used as testbeds to validate our experimental approach and interpretation.

In Fig. S2 we report the response of uniformly aligned $5 \mathrm{CB}$ and CCN47 cells to the turning on and off of the pump field for different values of the pump power (colored lines) in Geometries 1 and 2 (S2 A1, S2 A2, S2 B) and the intensity measured on the position of the first self-diffraction order for different values of $\mathrm{I}_{\mathrm{p}}$ (colored lines) in the case of CB7CB for $\mathrm{T}=106^{\circ} \mathrm{C}$ in Geometry $3(\mathrm{~S} 2 \mathrm{C}$ ). Dashed lines are best fits with single exponential functions (Fig. S2 A1, A2 and C) and double exponential functions with the constraint $\tau_{22}=\tau_{21} / 4$ (Fig. S2 B).
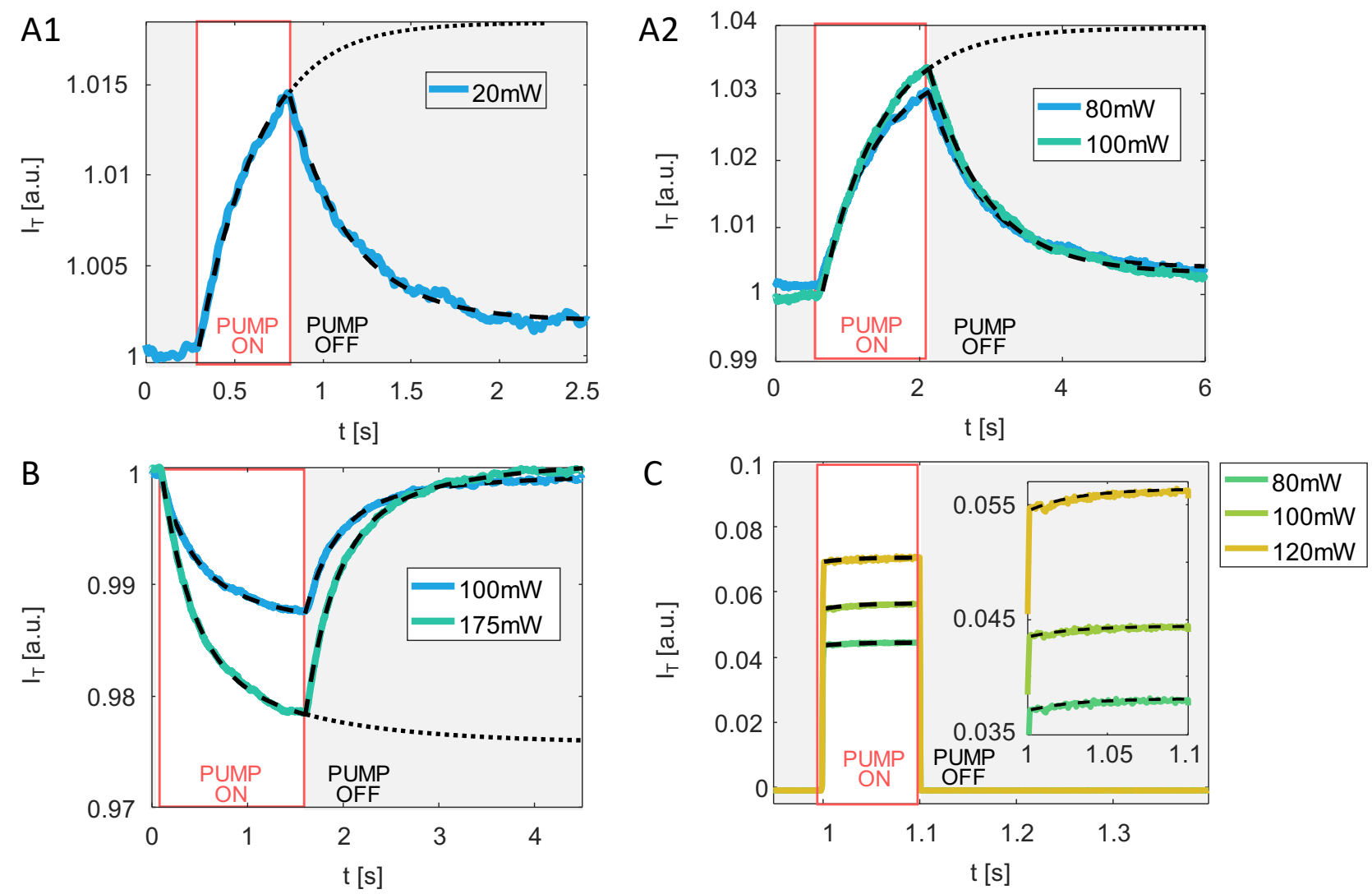

Figure S2. A1, A2, B: measured probe intensity vs time for a $13 \mu \mathrm{m}$ thick $5 \mathrm{CB}$ planar cell (A1) and for a $21 \mu \mathrm{m}$ thick $C C N 47$ planar cell (A2) in case of Geometry 1, and for a $10 \mu \mathrm{m}$ thick CCN 47 planar cell (B) in case of Geometry 2 for $\alpha=55^{\circ}$. C: self-diffracted intensity vs time for $C B 7 C B$ at $T=102^{\circ} \mathrm{C}$. Colored lines: data for different pump intensity as in legend. Dashed black lines: exponential (A1, $A 2$ and $C$ ) and double exponential (B) fitting lines, enabling determining the viscoelastic ratios in Table 1 of the main text. The extrapolated values for long times of the fitting functions (exemplified by the dotted lines) give $I_{i}, I_{2}$ and $I_{a}$, whose dependence on $I_{p}$ are shown in Figs. 2. A5, 2. B5 and 2. C5 of the main text, respectively. 


\section{Measuring twist elasticity and viscosity in chiral AAT}

As an additional validation of the results obtained for $\mathrm{K}_{2}$ and for the rotational viscosity $\eta_{\text {wisi }}$, we measured these two parameters also in chiral nematics of AAT. To do this, the "natural" right-handed selfcomplementary DNA oligomer 5'-AATGAATTCATT-3' and its enantiomer have been unbalanced in order to obtain chiral nematic LC with pitch $\mathrm{p}$ in the range $(2-3) \mu \mathrm{m}$. Measurements were performed on cells in planar geometry, where the chiral axis develops along $\mathrm{z}$, the direction normal to the cell surfaces. Worthy of note, such a geometry is spontaneously obtained in chiral DNA LCs.

The twist elastic coefficient has been measured by using the approach described in Ref. 11 of the main text previously applied on a chiral DNA LC. We refer the reader to that paper for a detailed description, while here we just give the fundamental information. Experiments were carried on with a pump-probe set up similar to the one described in the main text, with the pump light normally incident on the cell surface, linearly polarized either along $\mathrm{x}$ or $\mathrm{y}$ and propagating along the axis of the cholesteric helix. As discussed in Ref. 11, in this geometry the main effect of the incident pump light is a director perturbation within each pitch period p. To determine the value of $\mathrm{K}_{2}$ we used the probe light transmitted by the cell under the action of the pump beam, to compute the induced birefringence as a function of the pump intensity. A linear fit of this curve allows evaluating the twist coefficient, according to a specifically developed model. With that approach we determined the twist elastic coefficient but not the associated viscosity. We here extend that approach by also considering the kinetic behavior. Namely we determined the relaxation time following the turning on and off of the pump beam by a best fit of the kinetic behavior.

Figure S3 A shows the probe intensity transmitted by the chiral AAT cell during the turning on and off of the pump field for different values of the pump power (colored lines). Dashed lines are the best fit of the relaxation kinetics. Worthy of note, the relaxation follows a double exponential decay with two characteristic times. According to Ref. 11 of the main text, the main effect of the incident pump light propagating along the cholesteric helix axis is a director perturbation within each pitch period. The characteristic time associated to the relaxation of such a perturbation is $\tau=\eta_{t w i s t} p^{2} / 4 \pi^{2} K_{2}$ [5]. We attribute this value to the faster decay, while we understand the longer one - longer by about one order of magnitude, as associated to some additional phenomenon reflecting the sliding of the LC director over the surfaces. Such a sliding is not unexpected since in this cell no aligning layer was used. Figure S3 B reports the induced birefringence $\Delta \mathrm{n}$ as a function of the pump intensity $I_{p}$ with the linear fit necessary to extract $K_{2}$, as detailed in Ref. 11 .
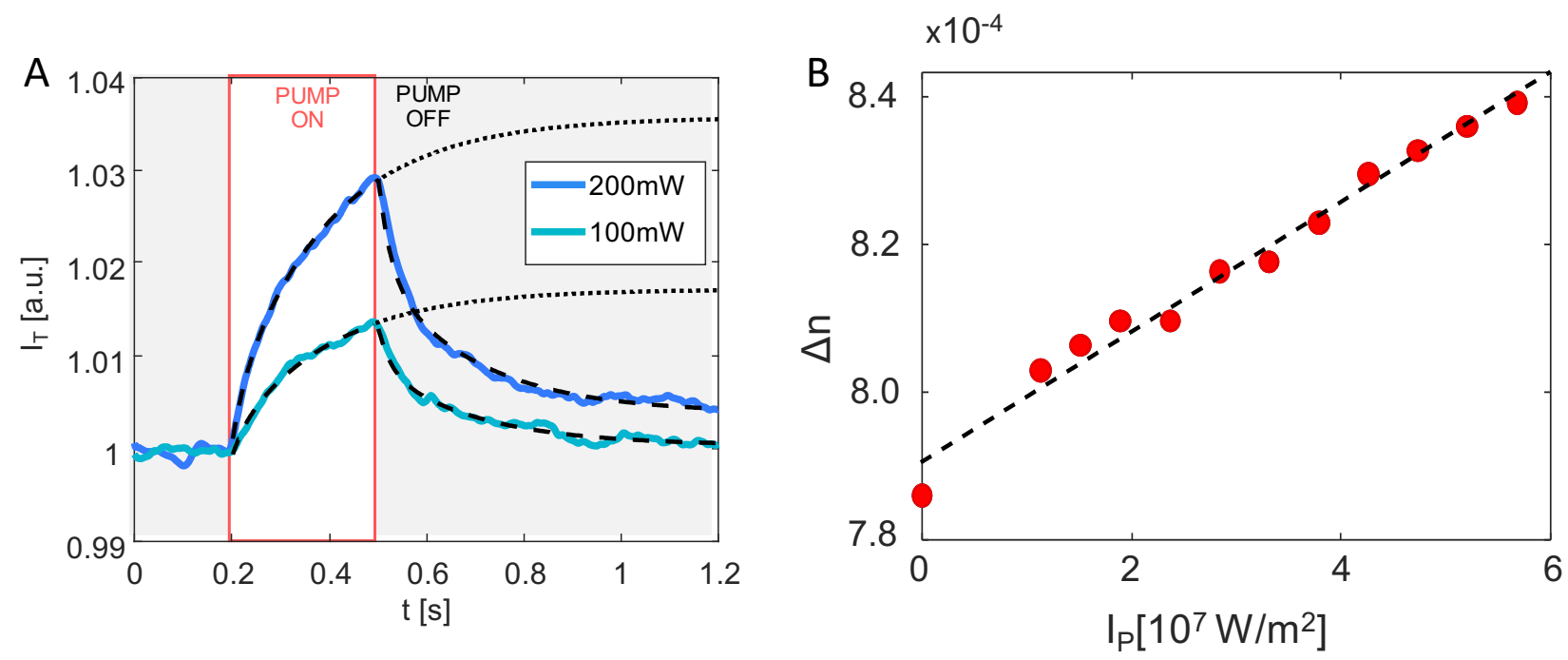

Figure S3: Measured probe intensity vs. time for two different values of the pump intensity (A). Dashed black lines: double exponential fitting lines, enabling determining the viscoelastic ratio in Table 1 of the main text. The extrapolated values for long times of the fitting lines (dotted lines) enable computing the induced birefringence as a function of $I_{p}$, displayed in $(B)$ with the linear fit from which $K_{2}$ is determined, as detailed in [10]. 


\section{References}

(1) Peng, C.; Guo, Y.; Turiv, T.; Jiang, M.; Wei, Q. H.; Lavrentovich, O. D. Patterning of Lyotropic Chromonic Liquid Crystals by Photoalignment with Photonic Metamasks. Adv. Mater. 2017, 29 (21), 1606112. https://doi.org/10.1002/adma.201606112.

(2) Yaroshchuk, O.; Reznikov, Y. Photoalignment of Liquid Crystals: Basics and Current Trends. $J$. Mater. Chem. 2012, 22 (2), 286-300. https://doi.org/10.1039/c1jm13485j.

(3) Yaroshchuk, O.; Kyrychenko, V.; Tao, D.; Chigrinov, V.; Kwok, H. S.; Hasebe, H.; Takatsu, H. Stabilization of Liquid Crystal Photoaligning Layers by Reactive Mesogens. Appl. Phys. Lett. 2009, 95 (2), 21902. https://doi.org/10.1063/1.3168526.

(4) Tseng, M.-C.; Yaroshchuk, O.; Bidna, T.; Srivastava, A. K.; Chigrinov, V.; Kwok, H.-S. Strengthening of Liquid Crystal Photoalignment on Azo Dye Films: Passivation by Reactive Mesogens. RSC Adv. 2016, 6 (53), 48181-48188. https://doi.org/10.1039/c6ra05298c.

(5) Zel'dovich, B. Y.; Tabiryan, N. V. Orientational Effect of a Light Wave on a Cholesteric Mesophase. J. Exp. Theor. Phys. 1982, 55 (1), 99. https://doi.org/10.1070/PU1985v028n12ABEH003985. 